\title{
Enhanced artemisinin yield by expression of rol genes in Artemisia annua
}

\author{
Erum Dilshad ${ }^{1}$, Rosa Maria Cusido², Javier Palazon², Karla Ramirez Estrada², Mercedes Bonfill ${ }^{2}$ \\ and Bushra Mirza ${ }^{1 *}$
}

\begin{abstract}
Background: Despite of many advances in the treatment of malaria, it is still the fifth most prevalent disease worldwide and is one of the major causes of death in the developing countries which accounted for 584,000 deaths in 2013, as estimated by World Health Organization. Artemisinin from Artemisia annua is still one of the most effective treatments for malaria. Increasing the artemisinin content of $A$. annua plants by genetic engineering would improve the availability of this much-needed drug.

Methods: In this regard, a high artemisinin-yielding hybrid of $A$. annua produced by the centre for novel agricultural products of the University of York, UK, was selected (artemisinin maximally $1.4 \%$ ). As rol genes are potential candidates of biochemical engineering, genetic transformation of A. annua with Agrobacterium tumefaciens GV3101 harbouring vectors with $\mathrm{rol} B$ and $\mathrm{rol} C$ genes was carried out with the objective of enhancement of artemisinin content. Transgenic lines produced were analysed by the LC-MS for quantitative analysis of artemisinin and analogues. These high artemisinin yielding transgenics were also analysed by real time quantitative PCR to find the molecular dynamics of artemisinin enhancement. Genes of artemisinin biosynthetic pathway were studied including amorphadiene synthase (ADS), cytochrome P450, (CYP71AV1) and aldehyde dehydrogenase 1 (ALDH1). Trichome-specific fatty acyl-CoA reductase 1 (TAFR1) is an enzyme involved in both trichome development and sesquiterpenoid biosynthesis and both processes are important for artemisinin biosynthesis. Thus, real time QPCR analysis of the TAFR1 gene was carried out, and trichome density was determined.

Results: Transgenics of rol $B$ gene showed two- to ninefold (the decimal adds nothing in the abstract, please simplify to two- to ninefold) increase in artemisinin, 4-12-fold increase in artesunate and 1.2-3-fold increase in dihydroartemisinin. Whereas in the case of rol $\mathrm{C}$ gene transformants, a fourfold increase in artemisinin, four to ninefold increase in artesunate and one- to twofold increase in dihydroartemisinin concentration was observed. Transformants with the $\mathrm{rol} B$ gene had higher expression of these genes than $\mathrm{rol} C$ transformants. TAFR1 was also found to be more expressed in rol gene transgenics than wild type $A$. annua, which was also in accordance with the trichome density of the respective plant.
\end{abstract}

Conclusion: Thus it was proved that $\mathrm{ro} B \mathrm{~B}$ and $\mathrm{rol} C$ genes are effective in the enhancement of artemisinin content of A. annua, rol $B$ gene being more active to play part in this enhancement than $\mathrm{rol} C$ gene.

Keywords: Artemisia annua, Artemisinin, rol genes, Hyb8001r, Trichomes

\section{Background}

Artemisinin, a highly potent anti-malarial agent found in the medicinal plant Artemisia annua, is part of the

\footnotetext{
*Correspondence: bushramirza@qau.edu.pk; dr.bushramirza@gmail.com

${ }^{1}$ Department of Biochemistry, Faculty of Biological Sciences, Quaid-i-

Azam University, Islamabad, Pakistan

Full list of author information is available at the end of the article
}

artemisinin-based combination therapy recommended by the World Health Organization for the treatment of malaria $[1,2]$. Besides malaria, artemisinin is also effective against some viral and parasitic diseases i.e. hepatitis $\mathrm{B}$, schistosomiasis, chagas disease and African sleeping sickness. It is also effective in the reduction of angiogenesis and treatment of some cancers [3]. Owing to the 
fact that availability of artemisinin is insufficient to meet worldwide demand, efforts are needed to increase its quantity. In this regard, some improvement has taken place by the Artemisia research project of CNAP (Centre for Novel Agricultural Products, University of York, UK). Their breeding programme has developed some high artemisinin-producing hybrids by making crosses between parental lines with consistent field performance, high artemisinin content and high seed productivity. Two hybrids which have been released commercially include Hyb1209r (Shennong, artemisinin $1.5 \%$ ) and Hyb8001r (Zenith, artemisinin 1.4\%) [4].

Artemisinin synthesis occurs in the glandular trichomes (GLTs) present on flowers, floral buds and leaves. Trichome-specific fatty acyl-CoA reductase 1 (TAFR1) is thought to be involved in GLT development and sesquiterpenoid biosynthesis; which are important for artemisinin production $[5,6]$. Other enzymes like amorphadiene synthase (ADS), cytochrome P450, CYP71AV1 (CYP), double bond reductase 2 (DBR2) and aldehyde dehydrogenase 1 (ALDH1) catalyze different steps of artemisinin biosynthesis [7-11]. Overexpression of these enzymes may lead to increased artemisinin accumulation and also the derivatives. Some studies provide evidence that metabolic engineering of the biosynthetic pathway that leads to artemisinin by the insertion of different genes can increase artemisinin content in planta $[12,13]$. In this regard, rol genes of $A$. rhizogenes are considered to be effective inducers of secondary metabolites production in plants [14]. Rol $A$ is a DNA binding protein and stimulator of growth, rol $B$ having tyrosine phosphatase activity regulates signal transduction pathway of auxin $[15,16]$, and rol $C$ has cytokinin glucosidase activity. However, each gene seems to affect plant morphology and stimulate production of different secondary metabolites [17-20]. However, there is no report about production of transgenic $A$. annua plants with individual rol genes for enhancement of artemisinin and its derivatives. Previous work on transformation of other Artemisia sp. with rol genes showed not only over expression of artemisinin biosynthetic genes, but also artemisinin in the plant [21].

In the continuation of that work, genetic transformation of $A$. annua (Hyb8001r, Zenith) with $\mathrm{rol}$ genes (rol B and $\mathrm{rol} C$ ) was carried out and consequences on the content of artemisinin were evaluated by LC-MS. The real time qPCR analysis of the genes involved in biosynthetic pathway of artemisinin (ADS, CYP71AV1 and ALDH1) provided an understanding of the molecular mechanism through which rol genes can enhance the artemisinin production. Expression of the gene for TAFR1 was also found to be relevant in this respect, providing evidence for molecular dynamics of artemisinin accumulation pattern and correlating it with the expression of genes involved in its biosynthesis.

\section{Methods}

\section{Seeds germination and DNA barcoding}

Seed of $A$. annua were purchased from East West seeds international company (Thailand). After collection and surface sterilization with $70 \%$ ethanol, they were germinated on half strength MS medium. Genomic DNA of germinated plantlets was extracted according to reported protocol [22]. Non-coding spacer region between the $p s b A$ and $t r n H$ genes of chloroplast DNA was amplified by PCR for the identification of $A$. annua, using primers of $p s b$ trnH region given in Table 1 . The PCR reaction was carried out according to the reaction conditions reported [23]. PCR products were purified by Rapid PCR Purification System 9700 (Marligen Biosciences, Ijamsville, MD, USA), which was then sequenced following dideoxy-chain termination method using an ABI Prism 310 Automated DNA Sequencer (PE, Applied Biosystems, Foster City, CA, USA). Sequences were identified and aligned via BioEdit sequence alignment tool (editor version 7.2.5.0).

\section{Bacterial strains and plasmids}

Agrobacterium tumefaciens strain GV3101 containing plasmid pPCV002-CaMVBT and pPCV002-CaMVC kindly provided by Dr. A. Spena, Max-Planck-Institut fur Zuchtungsforschung, 5000 Koin 30, FRG [24] were used for transformation purpose. The T-DNA region of the plasmid pPCV002-CaMVBT contained the coding sequence of rol $\mathrm{B}$ gene and that of pPCV002-CaMVC contained coding region of rol $\mathrm{C}$ gene, which were under the control of CaMV35S promoter. T-DNA of pPCV002CaMVBT and pPCV002-CaMVC also contained the neomycin phosphotransferase (NPTII) gene with nopaline synthase (NOS) promoter and NOS terminator sequences (Fig. 1). A. tumefaciens containing the plasmids pPCV002-CaMVBT and pPCV002-CaMVC were grown overnight in Luria broth (Sigma Cat \# L-1900). After inoculation, bacterial cultures were maintained at $28{ }^{\circ} \mathrm{C}$ and $120 \mathrm{rpm}$ in a shaking incubator. Growth was obtained in $24 \mathrm{~h}$ and OD was checked by spectrophotometer at $600 \mathrm{~nm}$, when in the range of $0.2-0.8$, the bacterial suspension was used for the transformation purpose.

Transformation and confirmation of transgene integration For transformation purpose 1 month-old in vitro grown plants were used. Nodal explants were prepared and after preculturing of 2 days on shooting media $(2.5 \mathrm{mg} / \mathrm{l}$ BAP and $0.25 \mathrm{mg} / \mathrm{l} \mathrm{NAA)} \mathrm{supplemented} \mathrm{with} 200 \mu \mathrm{M}$ acetosyringone, they were given the infection with bacterial strains containing the desired constructs. Explants 
Table 1 Accession numbers and primer sequences of the genes studied

\begin{tabular}{|c|c|c|c|}
\hline S. no & Gene name & Primer sequences & Accession no \\
\hline 1 & psbA-trnH & $\begin{array}{l}\text { Forward: GTTATGCATGAACGTAATGCTC-3 } \\
\text { Reverse: CGCGCATGGTGGATTCACAATC }\end{array}$ & FJ418749 \\
\hline 2 & Rol B & $\begin{array}{l}\text { Forward: GCTCTTGCAGTGCTAGATTT } \\
\text { Reverse: GAAGGTGCAAGCTACCTCTC }\end{array}$ & X15952.1 \\
\hline 3 & Rol C & $\begin{array}{l}\text { Forward: GAAGACGACCTGTGTTCTC } \\
\text { Reverse: CGTTCAAACGTTAGCCGATT }\end{array}$ & X64255.1 \\
\hline 4 & ADS & $\begin{array}{l}\text { Forward: ATTACTGGCGGTGCTAAC } \\
\text { Reverse: GTGCAGAGACAGCCCATT }\end{array}$ & JQ319661 \\
\hline 5 & CYP71AV1 & $\begin{array}{l}\text { Forward: ATTTTGGATATGTTTGGAGCAGGC } \\
\text { Reverse: TCCGCTTGTACTTTCTCCATTGCT }\end{array}$ & HQ315834 \\
\hline 6 & $\mathrm{ALDH} 1$ & $\begin{array}{l}\text { Forward: CAGGAGCTAATGGAAGTTCTAAGTCAG } \\
\text { Reverse: TTTCTTCCTTCGGCCACTGTTG }\end{array}$ & FJ809784 \\
\hline 7 & TFAR1 & $\begin{array}{l}\text { Forward: CCTTGGAGATCCTGAAGCTG } \\
\text { Reverse: CGTTGGATTGTGCTGAACTG }\end{array}$ & GU733320 \\
\hline 8 & Actin & $\begin{array}{l}\text { Forward: ATCAGCAATACCAGGGAACATAGT } \\
\text { Reverse: AGGTGCCCTGAGGTCTTGTTCC }\end{array}$ & EU531837 \\
\hline 9 & GADPH & $\begin{array}{l}\text { Forward:TGGGATCGTTGAGGGTCTT } \\
\text { Reverse: GCTGCTGGGAATAATGTTGAA }\end{array}$ & GQ870632.1 \\
\hline
\end{tabular}

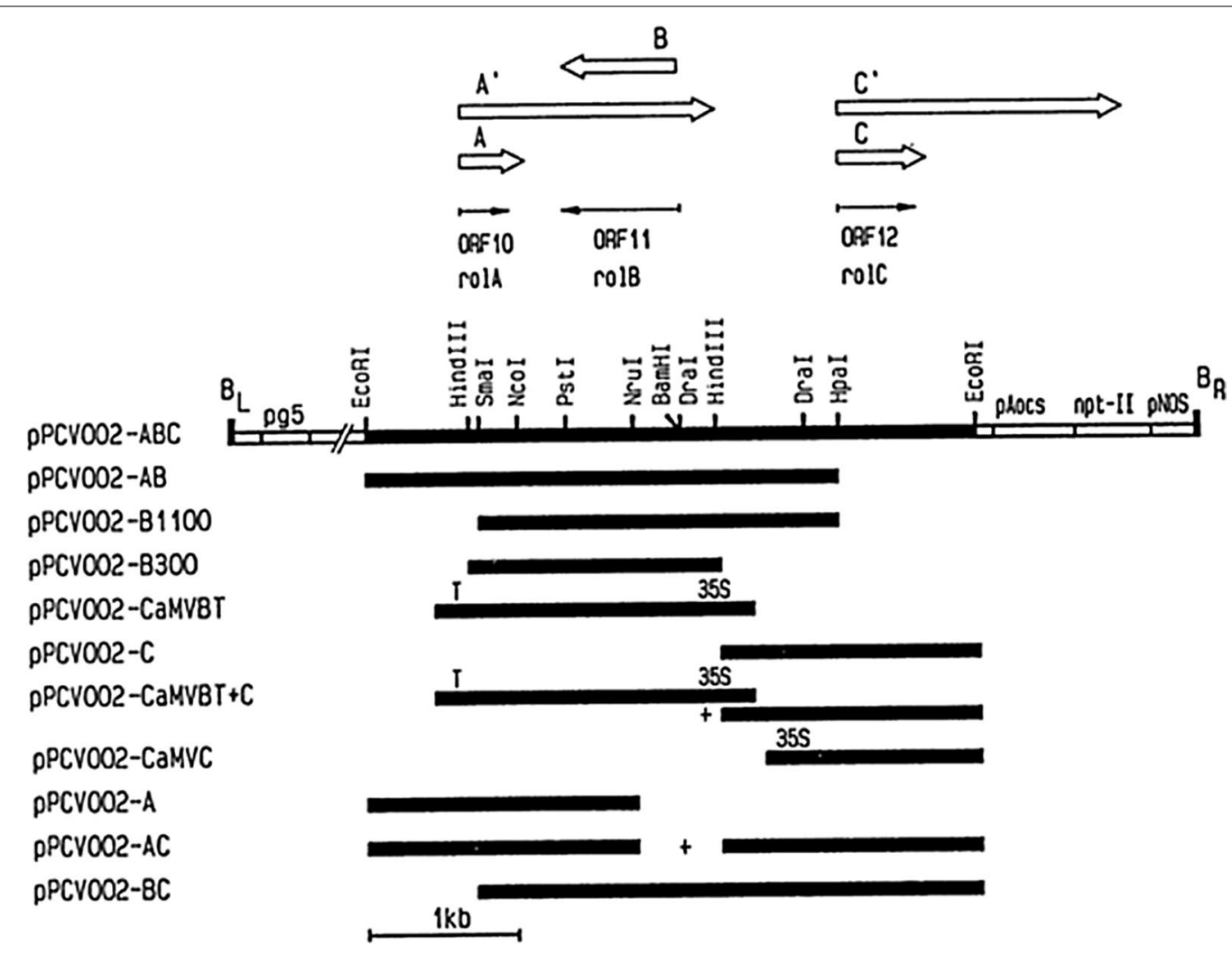

Fig. 1 Detail of vectors used for transformation of Artemisia annua: Vectors (pPCV002-CaMVBT and pPCV002-CaMVC) used in the transformation of Artemisia annua (Fig. taken from Spena et al. [24])

were given bacterial infection for 10-15 min afterwards explants were dried on autoclaved filter paper for 2-3 min and then placed on MS shooting media supplemented with $200 \mu \mathrm{M}$ acetosyringone. After 2 days of incubation in dark at $28{ }^{\circ} \mathrm{C}$, explants were washed with antibiotics and placed on selection medium $(2.5 \mathrm{mg} / \mathrm{l}$ 
BAP, $0.25 \mathrm{mg} / \mathrm{l} \mathrm{NAA}, 50 \mathrm{mg} / \mathrm{l}$ kanamycin, $300 \mathrm{mg} / \mathrm{l}$ cefotaxime). Regeneration occurred within 1 month, explants were shifted to fresh selection media after every 20 days. After getting the shoots of considerable, length they were shifted to the rooting media $(0.1 \mathrm{mg} / \mathrm{l} \mathrm{NAA}, 50 \mathrm{mg} / \mathrm{l} \mathrm{kan}$ amycin) Three to four cycles of selection were completed until complete plant was regenerated on selection media.

Molecular analysis was performed after extraction of genomic DNA from shoots of 2 month-old transformed and wild type plants by the CTAB method [22]. The plasmid from GV3101 was isolated by alkaline lysis method. PCR analysis was performed using a programmed DNA thermal cycler (Biometra, USA). The primers of $\operatorname{rol} B$ and $\mathrm{rol} C$ gene are given in Table 1. Conditions applied for PCR were according to a previous report [21].

\section{Analysis of artemisinin and its derivatives by HPLC-ESI/MS}

Extraction of artemisinin and its derivatives from leaves of 4-5 month old transformed and wild type plants was carried out with a previously published method [25]. HPLC-MS analysis was performed with Varian 500 Ion Trap Mass spectrometer (Varian, Spain) coupled to a HPLC system equipped with auto sampler (Varian, Spain). Separation of the analytes was achieved by $\mathrm{C} 18$ $(5 \mu \mathrm{m})$ column $(150 \mathrm{~mm} \times 4.6 \mathrm{~mm})$ (AKDAY Chromatografica, Spain) using mobile phase consisting of water with $0.1 \%$ formic acid (A) and acetonitrile (B) in a gradient as follows: [t $(\mathrm{min}), \% \mathrm{~B}](0,50)(10,50)(15,99)(23$, $50)(26,50)$. The flow rate was $1 \mathrm{ml} / \mathrm{min}$. Electrospray ionization (ESI) in positive mode was applied with a capillary voltage of $4000 \mathrm{~V}$ and plate voltage of $600 \mathrm{~V}$. Other parameters include: spray chamber temperature $50{ }^{\circ} \mathrm{C}$, nebulizing gas $\left(\mathrm{N}_{2}\right)$ pressure: $35 \mathrm{psi}$, dry gas $\left(\mathrm{N}_{2}\right) 10 \mathrm{~L} /$ min at $35 \mathrm{psi}$ and $400{ }^{\circ} \mathrm{C}$. The mass analyzer scanned from 66 to $500 \mathrm{u}$. Fragmentation amplitude was $1.0 \mathrm{~V}$.

\section{Expression analysis of rol genes and the genes related to artemisinin biosynthesis}

Total RNA from leaves of transformed and wild type plants (4-5 months old) was extracted according to a previously published procedure [26]. Turbo DNAse (Ambion) was used to ensure complete removal of DNA from RNA after extraction. The purity and quantity was checked by taking absorbance at 260 and $280 \mathrm{~nm}$ on Nanodrop ND-2000 spectrophotometer (Thermoscientific). The quality of RNA was also assessed by running RNA samples on $1.2 \%$ agarose gel. Reverse transcription of $1 \mu \mathrm{g}$ of RNA was carried by using 1st strand cDNA synthesis kit (Invitrogen) following the manufacturer's instructions. In order to check the expression of $r o l \mathrm{~B}$ and rol $\mathrm{C}$ gene, semi-quantitative reverse transcriptase-polymerase chain reaction was performed with $r o l \mathrm{~B}$ and $r o l$ $\mathrm{C}$ gene primers as done previously by using $1 \mu \mathrm{l}$ of cDNA reaction mixture as template. The gel image of PCR products were scanned by Kodak Molecular Imaging software (version 4.2) and integrated density values were calculated which were found to be different for each band.

To evaluate the possible effects of rol genes on the expression of artemisinin biosynthetic genes, quantitative real time PCR of four selected genes was performed, namely those encoding amorpha-4, 11 diene synthase (ADS), cytochrome P450 (CYP71AV1) and aldehyde dehydrogenase 1 (ALDH1). The gene involved in trichome development and sesquiterpenoid biosynthesis (TFAR1) was also studied. The $\beta$-actin gene was used as reference gene [27]. For real time qPCR, a 1:4 dilution of cDNA was used. The amplification reaction was performed by gene specific primers as given in Table 1; primer amplification efficiency was determined as previously described [28]. The qPCR was performed using iTAqTM Universal SYBR Green Supermix (BioRad, Hercules, CA, USA) in a 384-well platform system (ABI Prism ${ }^{\circledR} 7900$ HT sequence detection system, Applied Biosystems, Foster, CA, USA). The reaction conditions for real time qPCR were as follows: denaturation for $5 \mathrm{~min}$ at $95{ }^{\circ} \mathrm{C}$, followed by 45 cycles each of denaturation for $10 \mathrm{~s}$ at $95^{\circ} \mathrm{C}$, annealing for $10 \mathrm{~s}$ at $60{ }^{\circ} \mathrm{C}$, followed by elongation for $10 \mathrm{~s}$ at $72{ }^{\circ} \mathrm{C}$. For each gene, the relative expression levels were normalized with respect to the wild-type plant (reference value $=1$ ).

\section{Calculation of trichome density}

The leaves of wild type and transformed plants of $A$. annua were selected for the calculation of trichome density. For that purpose the ventral surfaces of the leaves were adhered to microscope slides with the help of glue stick and placed overnight at $4{ }^{\circ} \mathrm{C}$ for dehydration. The next day, slides were examined under the fluorescent microscope (Leica) with a FITC green filter. Slides were studied under $10 \times$ resolution and photographed.

\section{Statistical analysis}

All the experiments including, PCR, LC-MS and trichomes analysis were run in triplicate. Results of metabolites content, real-time quantitative PCR analysis and calculation of trichome density are presented as mean values $\pm \mathrm{SE}$. All the data obtained for the qualitative and quantitative analysis of artemisinin and derivatives, were statistically analysed by ANOVA and Duncan's multiple range test using Mstat $C$ software. Statistical significance of trichomes was determined by $\mathrm{t}$ test (**, $\mathrm{P}<0.01$; *, $\mathrm{P}<0.05)$.

\section{Results and discussion}

\section{Plant identification through DNA barcoding}

Amplification of $500 \mathrm{bp} p s b A-\operatorname{trn} H$ region of the chloroplast genome was successfully carried out. DNA samples 
were sequenced in triplicate to confirm the authenticity of species-specific nucleotides and got the same results. Gene bank accession number [NCBI: FJ418749] (A. annua psbA-trnH sequence) are reference sequence to confirm the plant species. After performing the CLUSTAL W in BioEdit software and the BLAST in NCBI, psbA-trnH sequence of $A$. аnnua was confirmed. There was $99.8 \%$ similarity between standard and analysed sequence with a single base pair difference at 330 bp position where cytosine was observed instead of adenine. Similar results were obtained by another group that amplified the $p s b A-t r n H$ region of $A$. annua under same conditions [23].

Transformation and confirmation of transgene integration Successful transformation of A. annua with A. tumefaciens GV3101 harbouring the rol B and rol C gene was carried out. Single transformation experiment was carried out, and 300 explants were used for each gene. Transformation efficiency was $30 \%$, calculated on the basis of plants showing PCR positive results with respect to total no of infected explants. Due to contaminating factors like fungal and bacterial, only three transformants of $\mathrm{rol} \mathrm{B}$ and three transformants of $\mathrm{rol} \mathrm{C}$ survived until maturity on selection media. PCR performed for the rol gene transformants of $A$. annua showed the amplified products of 779 bp for $r o l \mathrm{~B}, 549 \mathrm{bp}$ for rol C gene, as shown in Fig. 2a, b. Similar amplified products were obtained from plasmid DNA of GV3101-CaMVBT and GV3101-CaMVC respectively. The genome of wild type plants did not contain these genes.

Morphological variability was distinct among transgenics and wild type $A$. annua. Rol $\mathrm{B}$ transgenics grew faster on the selection media with altered morphology showing increased inflorescence, while transgenics of $\mathrm{rol} \mathrm{C}$ gene had totally different morphology with an altered leaf phenotype showing narrow leaf blade and decreased internode length (Fig. 3). It is reported that morphological variations can be due to the presence of rol genes transcripts and can be attributed to the position effect [29]. Several papers report variation in morphological characteristics of various plants species due to $\mathrm{rol}$ gene integration [30-32]. In fact, enhanced sensitivity of rol $B$ transformed cells toward auxin caused by increased tyrosine phosphatase activity of $\operatorname{rol} B$ gene can result in altered phenotype of rol $B$ transgenics [33, 34]. Apart from this, $\mathrm{H}^{+}$ATPase and ionic imbalance [33] also synchronize the metabolic pathways leading to phenotypic variations [35]. $\mathrm{Rol} \mathrm{C}$ is involved in functional imbalance of polyamines, gibberellins, auxins, and cytokinins [36,37]. Such imbalances in plant growth hormones can alter plant root and shoot phenotypes [35]. However, there was no effect on the overall growth rate of shoots as confirmed by measuring the fresh and dry weight of the wild type and transformed plants.

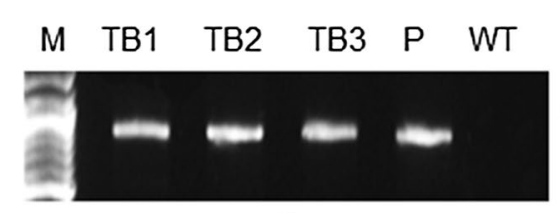

a
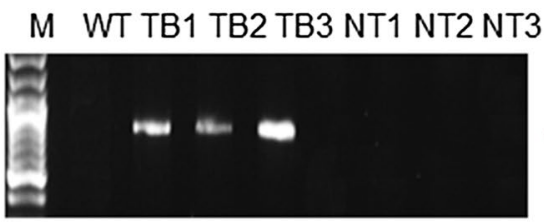

C

M TB1TB2 TB3 TC1 TC2TC3 WT NC

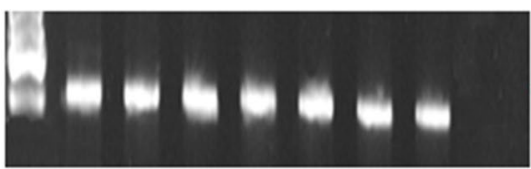

e

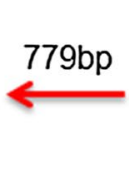

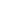

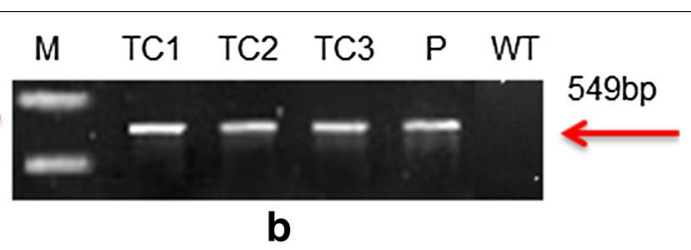

$M \quad W T$ TC1 TC2 TC3 NT1 NT2 NT3

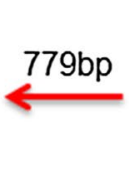

M TB1TB2 TB3TC1TC2TC3 WT NC

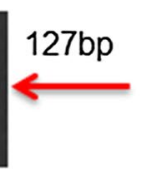

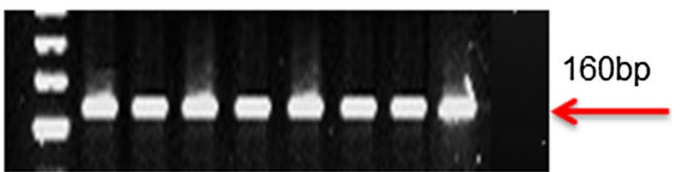

f

Fig. 2 Gel pictures showing results of normal and semiquantitative reverse transcriptase PCR: PCR amplified products of rol B (a), rol C (b) gene are shown in the figure. Semiquantitative RT-PCR showing the relative expression of rol B (c) and rol C gene (d). GADPH and $\beta$-actin used as housekeeping gene (e, f). TB1-TB3 represents rol $\mathrm{b}$ transgenics whereas TC1-TC3 represent rol $\mathrm{C}$ transgenics. WT stands for wild type plant of $A$. annua, lane $P$ refers to the plasmid DNA and lane $M$ corresponds to the marker DNA (Fermentas). NT indicates the negative or "no RT control" for each transgenic line 


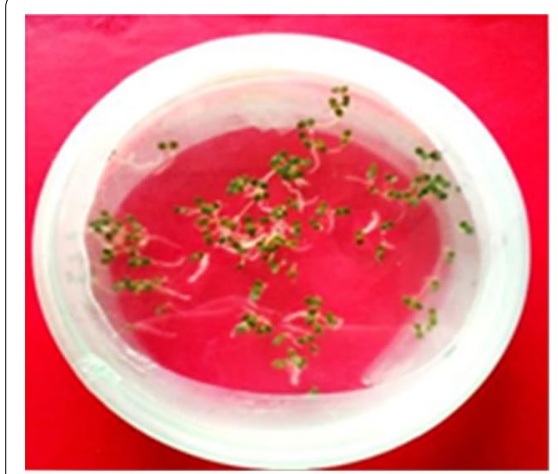

a

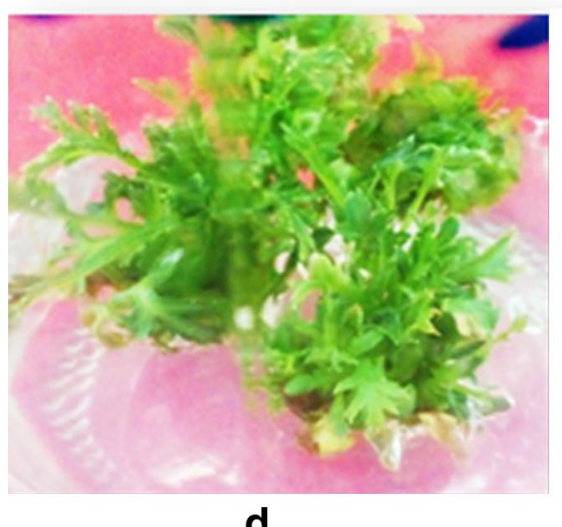

d

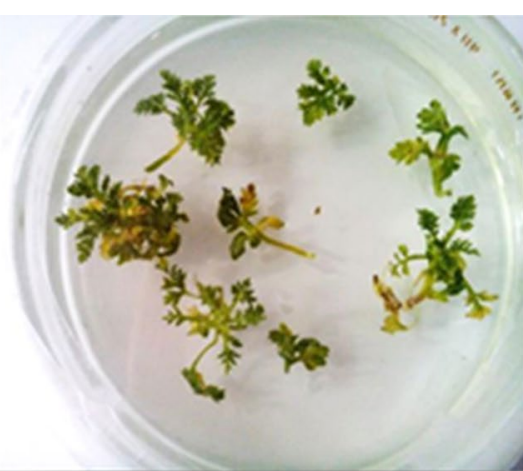

b

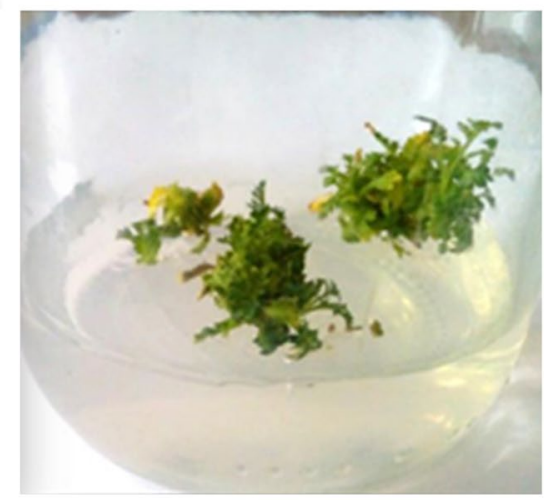

e

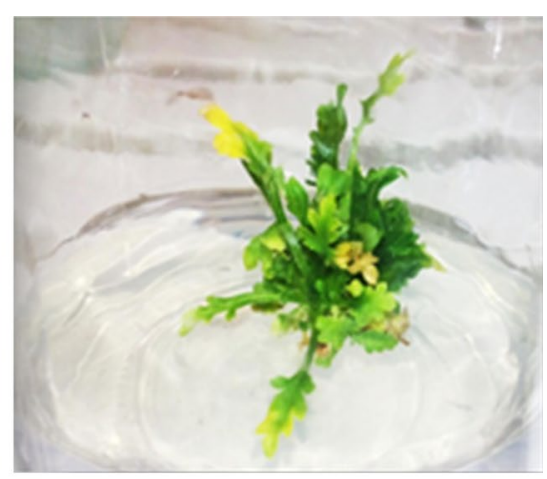

C

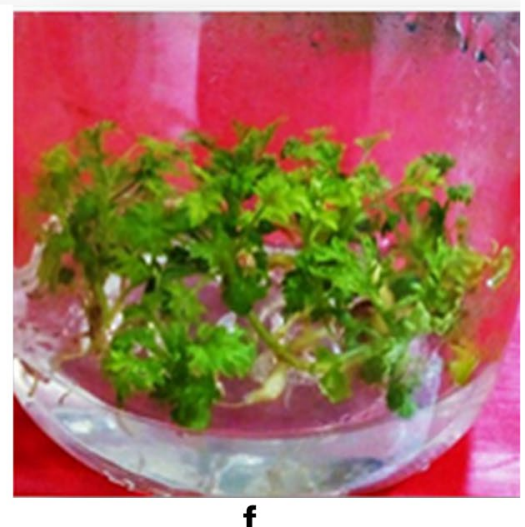

Fig. 3 Vegetative propagation of Artemisia annua: seeds germination (a), explants after co-cultivation with bacteria (b), regeneration on selection media (c), tranformant of rol B (d), transformant of rol C gene (e) and wild type A. annua (f)

\section{Analysis of artemisinin and derivatives by LC-MS}

Differences were observed in the content of artemisinin and derivatives in transformed and untransformed plants. All of the transgenic lines of $\mathrm{rol} B$ and $\mathrm{rol} C$ gene showed enhancement of artemisinin and derivatives (Fig. 4). Transgenics of $\mathrm{rol} \mathrm{B}$ gene showed 2.7-9.2-fold increase in artemisinin, 4-12.6-fold increase in artesunate and 1.2-3-fold increase in D.H.A. Whereas in case

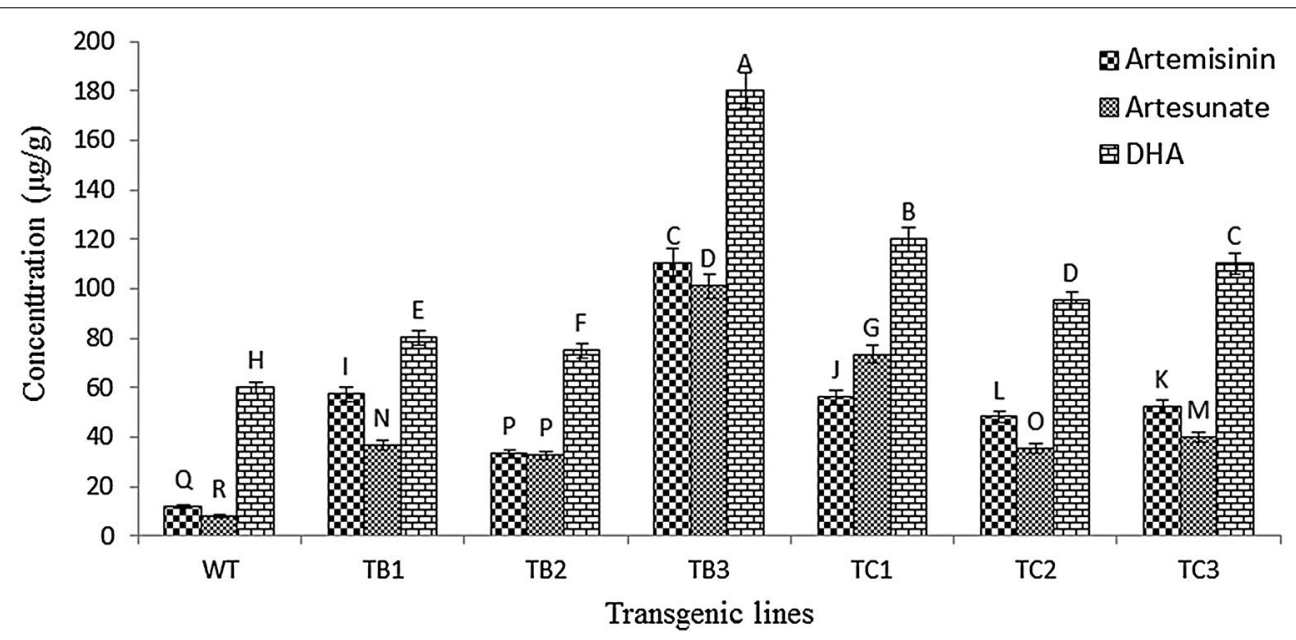

Fig. 4 Statistical and quantitative analysis of artemisinin and derivatives: comparative and statistical analysis of artemisinin content and its derivatives in wild type $A$. annua and transgenics of rol $B$ and rol $C$ gen. Letters on the bars show LSD ranking 
of rol $C$ gene transformants 4-4.6-fold increase in artemisinin, 4.4-9.1-fold increase in artesunate and 1.5-2fold increase in D.H.A concentration was observed. Previously it is reported that $\operatorname{rol}$ genes $(\operatorname{rol} A, \operatorname{rol} B$ and rol C) when expressed individually or combined result in increased plant secondary metabolism by transcriptional activation of defense genes through an unknown mechanism [38]. The effects of individual rol genes of TL-DNA of Agrobacterium rhizogenes, A4 strain on production of ginsenoside of Panax ginseng cell cultures has been reported [17]. In this report, rol $C$ cultures accumulated
1.8-3 times more ginsenoside than control plant [17]. Rol $B$ transgenics of Rubia cordifolia showed enhanced production of anthraquinones [38]. Further, rol $B$ gene from $A$. rhizogenes when expressed in tomato improved foliar tolerance against fungal pathogens [39]. However, in the current study differences in artemisinin content of $\mathrm{rol} \mathrm{B}$ and rol $C$ transgenics were observed. Rol $B$ gene was found to be more active in the enhancement of artemisinin content than $\mathrm{rol} C$ gene. As previously reported, high expression of the $\operatorname{rol} B$ gene dramatically increased the biosynthesis of secondary metabolites in transformed

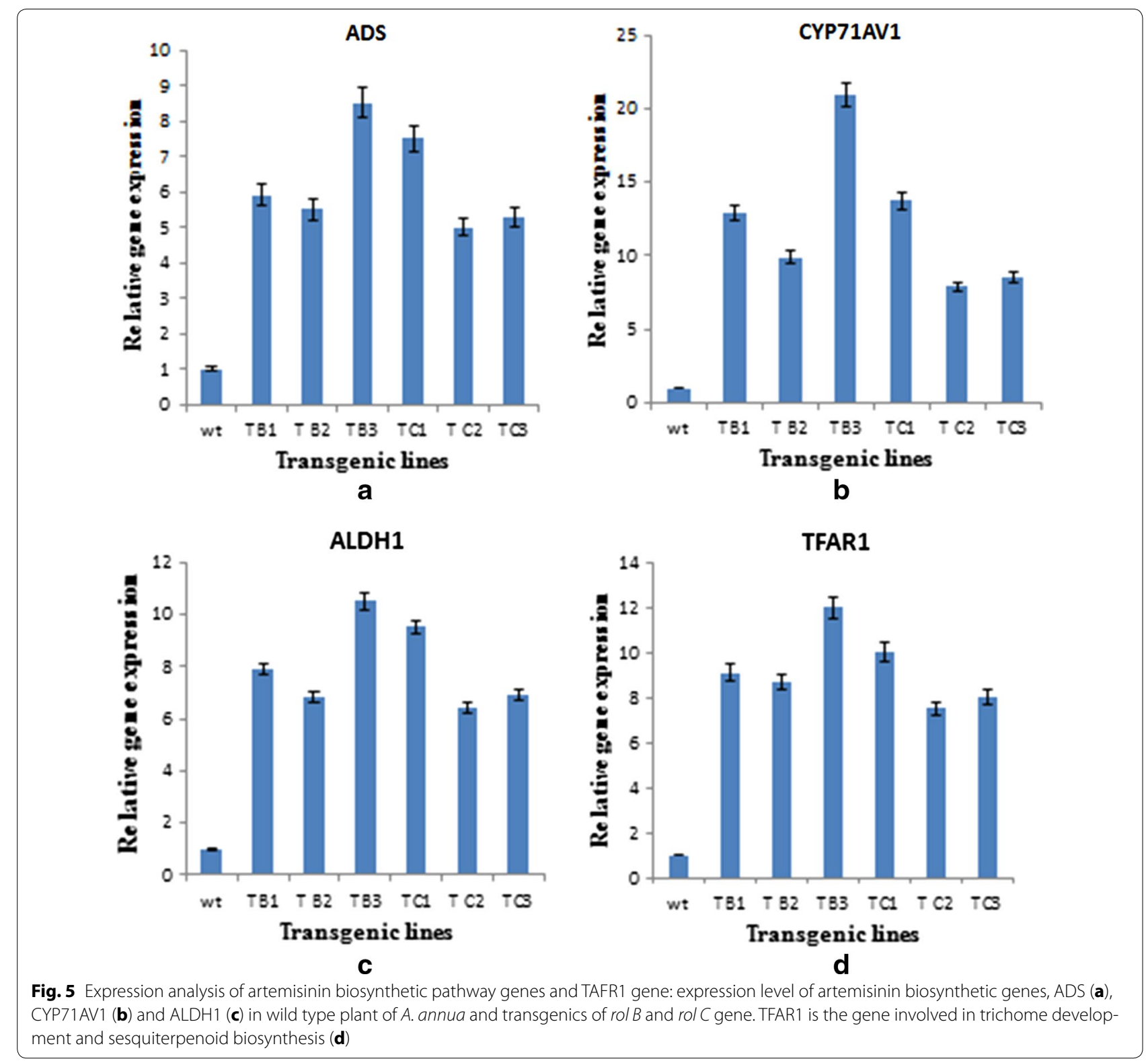


plant cells [38]. Compared to the $\operatorname{rol} B$ gene, the rol $C$ gene activated the biosynthesis of secondary metabolites to a lesser extent. Evidence indicates that each of the rol genes has its own role in plant metabolic processes [14]. Transgenic plants showing increased artemisinin concentration and altered morphology were analysed by semi quantitative RT-PCR to check that whether the results correlate with rol gene expression. Results confirmed the findings to be the result of presence of rol gene transcripts. Also it was observed that the lines TB3 and TC1 had higher artemisinin content and more transcripts of rol $\mathrm{B}$ and rol $\mathrm{C}$ gene, respectively (Fig. 2c, d). As these genes are under the control of CaMV35S promoter, their effect on the induction of secondary metabolism become more clear due to systemic high level of expression [40].

\section{Expression analysis of artemisinin and trichome biosynthesis genes}

Relatively higher transcripts of all studied genes of artemisinin biosynthetic pathway were found in the transgenics of $r o l \mathrm{~B}$ and $\mathrm{rol} \mathrm{C}$ gene although in variable fashion (Fig. 5), which also correlates the accumulation pattern of artemisinin and analogues. Regarding the biosynthesis of artemisinin, ADS, CYP71AV1 and ALDH1 are key genes involved in the conversion of FDP (Farnesyl diphosphate) to artemisinic acid which is a late precursor of artemisinin, as discussed in the background section [7-11]. Transgenics of $\mathrm{rol} \mathrm{B}$ and $\mathrm{rol} C$ gene showed overexpression of studied genes with CYP71AV1 found to be most expressed in transgenics with 8-20.9-fold increase in rol B tranformants and 6-15.5-fold more expression in rol C transformants. ALDH1 gene showed eight- to tenfold and ADS gene showed 5.9-8.5-fold more expression in rol B transformants. Similarly Transgenics of rol $C$ gene showed 6.4-9.5-fold increase in ALDH1 and 5-7.5-fold increase in ADS gene expression. Transgenic line TB3 and TC1 were found with highest expression of these genes. This relative expression of all the genes is in accordance with the accumulation of artemisinin and derivatives in respective transgenic lines. These results are supported by a previous report which demonstrates that overexpression of farnesyl pyrophosphate synthase (FPS) in $A$. annua increases the accumulation of artemisinin through conversion of isopentenyl diphosphate (IPP) and dimethylallyl diphosphate (DMADP) into farnesyl diphosphate (FDP) [41]. In another report, increased amount of artemisinin in $A$. annua transformed with A. tumefaciens wild type nopaline strains was observed [42]. Similarly, another group transferred the Ipt gene into A. annua and observed increased amount of artemisinin in transformed plants [13].

\section{Trichome density and expression of TFAR 1 gene}

The number of glandular trichomes was determined at the adaxial side of three random pieces of fresh leaf material from each sample with an accurately determined leaf area of approximately $1 \mathrm{~cm}^{2}$ per leaf and significant differences were observed as shown by Fig. 6 .
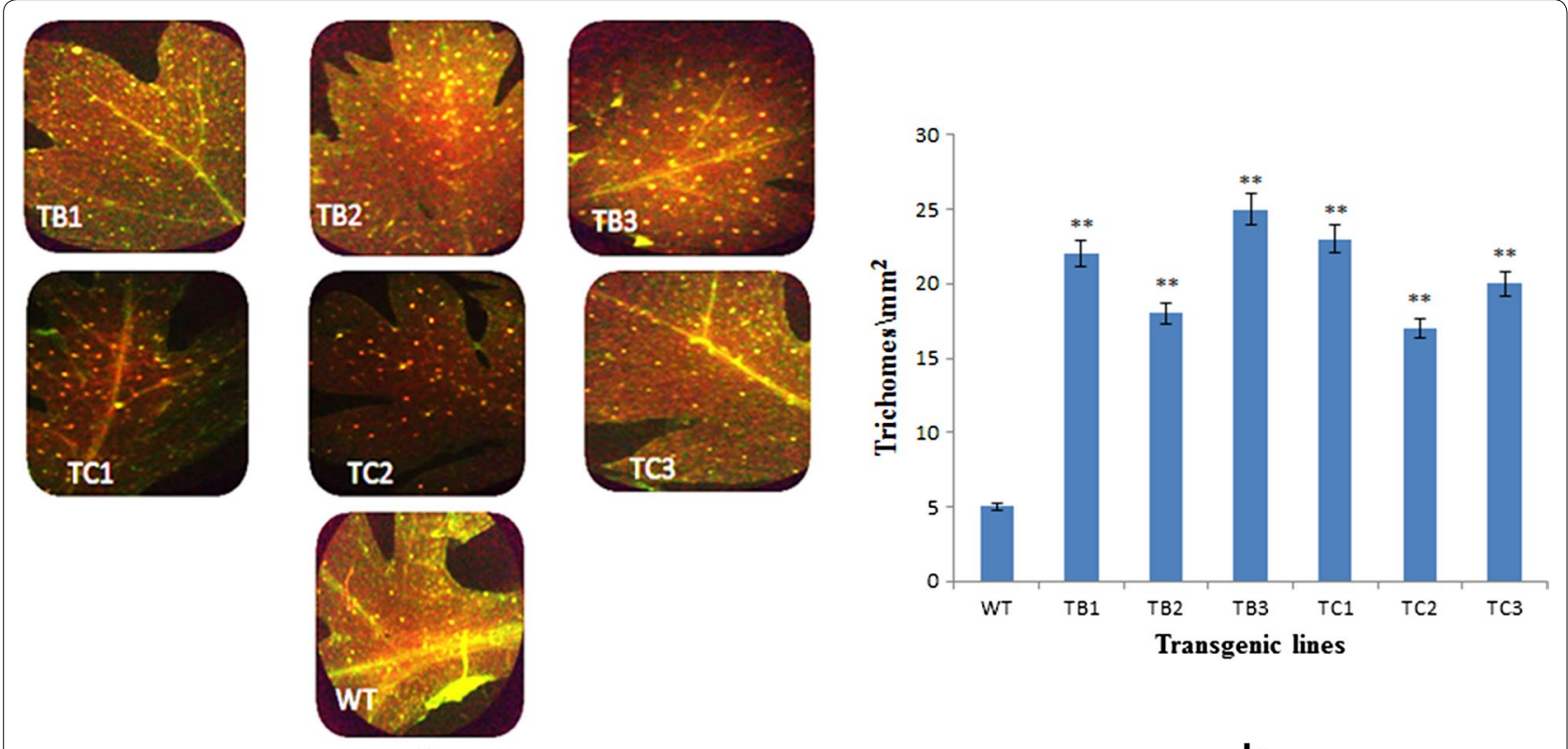

a

\section{b}

Fig. 6 a Trichome density: comparison of trichome density of transformed and untransformed plants of $A$. annua. TB1-TB3 represent rol $B$ transgenics whereas TC1-TC3 represent transgenics of rol C gene. WT represents the control plant of A. annua. $\mathbf{b}$ Graphical representation of trichomes density of wild type $A$. annua and transformants of $r o l B$ and $r o l C$ gene 
The leaves of $A$. annua transformed with $\operatorname{rol} B$ and $\operatorname{rol} C$ gene produced more trichomes $\left(20-30\right.$ trichomes $\left./ \mathrm{cm}^{2}\right)$ as compared to untransformed leaves (5-7 trichomes/ $\mathrm{cm}^{2}$ ).

TFAR1, which stimulates trichome development and catalyzes sesquiterpenoid biosynthesis [41] also showed increased expression in all transgenic lines. Rol B transformants with 2.3-3-fold increase and rol C gene transformants with 1.6-2-fold increase in TFAR1 gene expression were found. As mentioned in a previous report that glandular trichomes are the sites of production of important phytochemicals including artemisinin and a mixture of chemicals that have been found to have an enormous array of uses in the pesticides, pharmaceutical and flavor industries $[43,44]$. Artemisinin content was found to be directly related to the trichome index in previous reports $[45$, 46].

\section{Conclusion}

Transformation of A. annua with rol B and rol C gene results in the enhancement of its secondary metabolites particularly the artemisinin and derivatives, $\operatorname{rol} B$ gene being more active to play part in the enhancement of artemisinin than $\mathrm{rol} C$ gene. Further the level of transcripts of the rol $B$ and rol $C$ gene found in transgenics also correlate with their artemisinin accumulation pattern. Altered expression of genes involved in biosynthesis of artemisinin and trichomes was observed.

\footnotetext{
Abbreviations

ADS: amorpha diene synthase; ALDH1: aldehyde dehydrogenase 1; CTAB: cetyl trimethylammonium bromide; CNAP: Centre for Novel Agricultural Products; CYP71AV1: cytochrome P450; DBR2: double bond reductase 2; D.H.A: dihydroartemisinin; DMADP: dimethyl diphosphate; FDP: farnesyl diphosphate; FPP: farnesyl pyrophosphate; GADPH: glyceraldehyde-3-phosphate dehydrogenase; GLTs: glandular trichomes; IPP: isomethyl diphosphate; TAFR1: trichome specific fattyacyl CoA reductase 1.
}

\section{Authors' contributions}

ED carried out the practical work regarding all experiments i.e. DNA barcoding, genetic transformation, molecular analysis, LC-MS and trichome study. RMC \& JP supervised all the work performed in the department of pharmacy, University of Barcelona Spain. They also reviewed the manuscript intellectually and critically to give it final form for publication. KR provided technical as well as intellectual support in doing real time quantitative PCR analysis. She also made efforts in making experimental design. MB supervised the quantitative analysis of artemisinin and derivatives by HPLC-MS by helping in experimental design and interpretation of results. BM supervised all the work performed in plant molecular Biology laboratory of QAU Islamabad Pakistan. She provided the intellectual support in making the experimental design of DNA barcoding, genetic transformation and trichome study. She also helped in writing the manuscript and its proofreading. All authors read and approved the final manuscript.
\end{abstract}

\section{Author details}

${ }^{1}$ Department of Biochemistry, Faculty of Biological Sciences, Quaid-i-Azam University, Islamabad, Pakistan. ${ }^{2}$ Laboratorio de Fisiologia Vegetal, Facultad de Farmacia, Universidad de Barcelona, Barcelona, Spain.

\section{Acknowledgements}

We are grateful to Dr. A. Spena, Max-Planck-Institut fur Zuchtungsforschung, 5000 Koin 30, FRG for providing the vectors. We are thankful to the Higher Education Commission (HEC) of Pakistan for financially supporting this research project. Part of this work was financially supported by the Spanish MEC (BIO2014-51861-R) and the Generalitat de Catalunya (2014SGR215).

\section{Competing interests}

The authors declare that they have no competing interests.

Received: 3 June 2015 Accepted: 21 October 2015

Published online: 29 October 2015

\section{References}

1. Duffy PE, Mutabingwa TK. Artemisinin combination therapies. Lancet. 2006;367:2037-9.

2. White NJ. Qinghaosu (artemisinin): the price of success. Science. 2008;320:330-4.

3. Efferth TW. Schwabe Award 2006: antiplasmodial and antitumor activity of artemisinin—from bench to bedside. Planta Med. 2007;73:299-309.

4. CNAP Artemisia annua F1 hybrids. 2015. http://www.york.ac.uk/org/cnap/ artemisiaproject/artemisiaF1seed/index.html. Accessed 15 May 2015.

5. Ferreira JFS, Simon JE, Janick J. Developmental studies of Artemisia annua: flowering and artemisinin production under greenhouse and field conditions. Planta Med. 1995;61:167-70.

6. Olsson ME, Olofsson LM, Lindahl AL, Lundgren A, Brodelius M, Brodelius PE. Localization of enzymes of artemisinin biosynthesis to the apical cells of glandular trichomes of Artemisia annua L. Phytochemistry. 2009;70:1123-8.

7. Brown GD, Sy LK. In vivo transformations of artemisinic acid in Artemisia annua plants. Tetrahedron. 2007;63:9548-66.

8. Croteau R, Kutchan T, Lewis N. Natural products (secondary metabolites). In: Buchanan B, Gruissem W, Jones R, editors. Biochemistry and molecular biology of plants. Rockville: American Society of Plant Physiology; 2000. p. 1250-318.

9. Teoh KH, Polichuk DR, Reed DW, Nowak G, Covello PS. Artemisia annua L. (Asteraceae) trichome-specific cDNAs reveal CYP71AV1, a cytochrome P450 with a key role in the biosynthesis of the antimalarial sesquiterpene lactone artemisinin. FEBS Lett. 2006;580:1411-6.

10. Wallaart TE, Pras N, Beekman AC, Quax WJ. Seasonal variation of artemisinin and its biosynthetic precursors in plants of Artemisia annua of different geographical origin: proof for the existence of chemotypes. Planta Med. 2000;66:57-62.

11. Zhang Y, Teoh KH, Reed DW, Maes L, Goossens A, Olson DJH, et al. The molecular cloning of artemisinic aldehyde D11 (13) reductase and its role in glandular trichome-dependent biosynthesis of artemisinin in Artemisia annua. J Biol Chem. 2008;283:21501-8.

12. Chen $\mathrm{D}, \mathrm{Ye} H, \mathrm{Li}$ G. Expression of a chimeric farnesyl diphosphate synthase gene in Artemisia annua L. transgenic plants via Agrobacterium tumefaciens-mediated transformation. Plant Sci. 2000;155:179-85.

13. Sa G, Ma M, Ye HC, Liu BY, Li GF, Chong K. Effects of ipt gene expression on the physiological and chemical characteristics of Artemisia annua L. Plant Sci. 2001;160:69198.

14. Bulgakov VP. Functions of $R O L$ genes in plant secondary metabolism. Biotechnol Adv. 2008;26:31824.

15. Filippini F, Lo Schiavo F, Terzi M, Constantino P, Trovato M. The plant oncogene rol B alters binding of auxin to plant membranes. Plant Cell Physiol. 1994;35:767-71.

16. Filippini F, Rasi V, Marin O, Trovato M, Downey PM, Lo SF, et al. A plant oncogene as a phosphatase. Nature. 1994;379:499-500.

17. Bulgakov VP, Khodakovskaya MV, Labetskaya NV, Tchernoded GK, Zhuravlev YN. The impact of plant rol C oncogene on ginsenoside production by ginseng hairy root cultures. Phytochemistry. 1998;49:1929-34.

18. Casanova E, Trillas MI, Moysset L, Vainstein A. Influence of rol genes in floriculture. Biotechnol Adv. 2005;23:3-39.

19. Palazon J, Cusido RM, Roig C, Pinol MT. Expression of the ro/ C gene and nicotine production in transgenic roots and their regenerated plants. Plant Cell Rep. 1998;17:384-90. 
20. Palazon J, Cusido RM, Gonzalo J, Bonfill M, Morales S, Pinol MT. Relation between the amount the ro/ $\mathrm{C}$ gene product and indole alkaloid accumulation in Catharanthus roseus transformed root cultures. J Plant Physiol. 1998;153:712-8.

21. Kiani BH, Safdar N, Mannan A, Mirza B. Comparative Artemisinin analysis in Artemisia dubia transformed with two different Agrobacteria harbouring rol ABC genes. Plant Omics J. 2012;5:386-91.

22. Ahmed I, Islam M, Arshad W, Mannan A, Ahmad W, Mirza B. Highquality plant DNA extraction for PCR: an easy approach. J Appl Genet. 2009;50:105-7.

23. Tao L, Yunheng J.p psbA-trnH sequence analysis from chloroplast on medicinal plants of Artemisia. Chin Agric Sci Bull. 2009;25:46-9.

24. Spena A, Schmiilling T, Koncz C, Schell JS. Independent and synergistic activity of rol $A, B$ and $C$ loci in stimulating abnormal growth in plants. EMBO J. 1987;6:3891-9.

25. Towler MJ, Weathers PJ. Evidence of artemisinin production from IPP stemming from both the mevalonate and the non-mevalonate pathways. Plant Cell Rep. 2007;26:2129-36.

26. Safdar N. Agrobacterium-mediated transformation of tomato with yeast halotolerance genes. Dissertation. Quaid-iAzam University Islamabad; 2012. p. 36-7.

27. Olofsson L, Engstrom A, Lundgren A, Brodelius PE. Relative expression of genes of terpene metabolism in different tissues of Artemisia annua $\mathrm{L}$. BMC Plant Biol. 2011;11:45-7.

28. Sabater-Jara AB, Onrubia M, Moyano E, Bonfill M, Palazon J, Pedreno $M A$, et al. Synergistic effect of cyclodextrins and methyl jasmonate on taxane production in Taxus $\times$ media cell cultures. Plant Biotech J. 2014; doi:10.1111/pbi.12214.

29. Schmulling T, Schell J, Spena A. Single genes from Agrobacterium rhizogenes influence plant development. EMBO J. 1988;7:2621-9.

30. Cardarelli M, Mariotti D, Pomponi M, Spano L, Capone I, Costantino P. Agrobacterium rhizogenes T-DNA genes capable of inducing hairy root phenotype. Mol Gen Genet. 1987;209:475-80.

31. Van Altvorst AC, Bino RJ, van Dijk AJ, Lamers AMJ, Lindhout WH, Van der Mark F, et al. Effects of the introduction of Agrobacterium rhizogenes $R O L$ genes on tomato plant and flower development. Plant Sci. 1992:83:77-85.

32. Spena A, Esttruch JJ, Prinsen E, Nacken W, Vanonckelen H, Sommer H. Anther-specific expression of the rolB gene of Agrobacterium-rhizogenes increases IAA content in anthers and alters anther development and whole flower growth. Theor Appl Genet. 1992;84:520-7.

33. Maurel C, Barbier-Brygoo H, Spena A, Tempe J, Guern J. Single ROL gene from the Agrobacterium rhizogenes TL-DNA alters some of the cellular responses to auxin in Nicotiana tabacum. Plant Physiol. 1991;97:212-6.
34. Maurel C, Leblanc N, Barbier-Brygoo H, Perrot-Rechenmann C, BouvierDurand M, Guren J. Alterations of auxin perception in rolB transformed tobacco protoplast. Plant Physiol. 1994;105:1209-15.

35. Zia M, Mirza B, Malik SA, Chaudhary MF. Expression of ROL genes in transgenic soybean (Glycine max L.) leads to changes in plant phenotype, leaf morphology, and flowering time. Plant Cell Tissue Organ Cult. 2010;103:227-36.

36. Cui M, Takayanagi K, Kamada H, Nishimura S, Handa T. Efficient shoot regeneration from hairy roots of Antirrhinum majus $L$. transformed by the rol type MAT vector system. Plant Cell Rep. 2001;20:55-9.

37. García-Sogo B B, Pineda B, Castelblanque L, Antón T, Medina M, Roque E, et al. Efficient transformation of Kalanchoe blossfeldiana and production of male-sterile plants by engineered anther ablation. Plant Cell Rep. 2010;29:61-77.

38. Shkryl YN, Veremeichik GN, Bulgakov VP, Tchernoded GK, Mischenko NP, Fedorevev SA, et al. Individual and combined effects of the rol A, B and C genes on anthraquinone production in Rubia cordifolia transformed calli. Biotechnol Bioeng. 2008;100:118-25.

39. Arshad W, Haq IU, Waheed MT, Mysore KS, Mirza B. Agrobacterium-mediated transformation of tomato with rol $B$ gene results in enhancement of fruit quality and foliar resistance against fungal pathogens. PLoS One. 2014;9:e96979. doi:10.1371/journal.pone.0096979.

40. Odell JT, Nagy F, Chua NH. Identification of DNA sequences required for activity of the cauliflower mosaic virus 355 promoter. Nature. 1985;313:810-2.

41. Lies M, Filip CW, Van N, Yansheng Z, Darwin WR, Jacob P. Dissection of the phytohormonal regulation of trichome formation and biosynthesis of the antimalarial compound artemisinin in Artemisia annua plants. New Phytol. 2011;189:176-89.

42. Biswajit G, Swapna M, Sumita J. Genetic transformation of Artemisia annua by Agrobacterium tumefaciens and artemisinin synthesis in transformed cultures. Plant Sci. 1997;122:193-9.

43. Duke MV, Paul RN, Elsohly HN, Sturtz G, Duke SO. Localization of artemisinin and artemisitene in foliar tissues of glanded and glandless biotypes of Artemisia annua L. Int J Plant Sci. 1994;155:365-72.

44. Ferreira JF, Simon JE, Janick J. Artemisia annua: botany, horticulture, pharmacology. In: Janick J, editor. Horticultural reviews. New York: John Wiley and Sons, Inc.; 1997. p. 319-71.

45. Dangash A, Pandya N, Bharillya A, Jhala A, Jain D. Impact of exogenous elicitors on artemisinin production and trichome density in Artemisia annua L. under subtropical conditions. Not Sci Biol. 2014;6:349-53.

46. Kiani BH. Transfer of rol genes and evaluation of artemisinin synthesis in transformed Artemisia annua L. and Artemisia dubia WALL. Dissertation. Quaid-i-Azam University Islamabad; 2013.

\section{Submit your next manuscript to BioMed Central and take full advantage of:}

- Convenient online submission

- Thorough peer review

- No space constraints or color figure charges

- Immediate publication on acceptance

- Inclusion in PubMed, CAS, Scopus and Google Scholar

- Research which is freely available for redistribution

Submit your manuscript at 